\title{
FIRST DENSITY COMPARISON ON VISCOELASTIC SAMPLES BY OSCILLATION-TYPE DENSIMETRY
}

\author{
A. Furtado ${ }^{1}$, J. Pereira ${ }^{2}$, R. Quendera ${ }^{3}$, M. Schiebl ${ }^{4}$, E. Lenard ${ }^{5}$, E. Malejczyk ${ }^{6}$, A. Alic ${ }^{7}$, S. Alisic $^{8}$, J Rauch $^{9}$, F \\ Lorenz $^{10}$, A. Bescupschii ${ }^{11}$, A. Ciubara ${ }^{12}$, B. Laky ${ }^{13}$, R. Amsüss ${ }^{14}$ \\ 1,2,3 Instituto Português da Qualidade (IPQ), Portugal, ${ }^{1}$ afurtado@ipq.pt, ${ }^{2}$ ipereira@ipq.pt, ${ }^{3}$ rquendera@ipq.pt \\ ${ }^{4}$ Physikalisch Technischer Prüfdienst des Bundesamt für Eich und Vermessungswesen (BEV-PTP), Austria, \\ Markus.Schiebl@bev.gv.at \\ 5, ${ }^{6}$ Central Office of Measures (GUM), Poland, ${ }^{5}$ elzbieta.lenard@ gum.gov.pl, ${ }^{6}$ ewa.malejczyk@gum.gov.pl \\ ${ }^{7,8}$ Institut za mjeriteljstvo Bosne i Hercegovine (IMBiH), Bosnia and Herzegovina, ${ }^{7}$ azmir.alic@ met.gov.ba, \\ 8 sejla.alisic@met.gov.ba \\ 9, 10 Physikalisch Technische Bundesanstalt (PTB), Germany, ${ }^{9}$ Juergen.Rauch@ptb.de, ${ }^{10}$ FLorenz@ptb.de \\ ${ }^{11,12}$ I.P. Institutul National de Metrologie (INM), Republic of Moldova, ${ }^{11}$ anatolii.bescupschii@inm.gov.md, \\ 12 ana.curdov@inm.gov.md \\ 13, 14 Anton Paar GmbH, Austria, ${ }^{13}$ barbara.laky@anton-paar.com, ${ }^{14}$ robert.amsuess@anton-paar.com
}

\begin{abstract}
:
To provide a deeper insight about the damping effects produced by the viscoelasticity of nonNewtonian fluids during density measurements with oscillation-type density meters, and about how reference laboratories overcome these effects, an international comparison was performed, within the scope of the EMPIR Project 17RPT02 rhoLiq.

The results of the comparison evidenced the possibility to measure density of viscoelastic samples by means of oscillation-type density meters with an uncertainty between $0.10 \mathrm{~kg} \cdot \mathrm{m}^{-3}$ and $0.25 \mathrm{~kg} \cdot \mathrm{m}^{-3}$. However, these instruments may be able to reach lower uncertainties if compared with higher precision density measurement methods such as hydrostatic weighing.
\end{abstract}

Keywords: density; viscoelasticity; oscillationtype densimetry: degree of equivalence; rhoLiq

\section{INTRODUCTION}

The measuring principle of an oscillation-type density meter is based on the law of harmonic oscillation [1]. In short, in these instruments, the measuring cell, that acts like a flexural oscillator, is filled with the fluid sample and is subjected to an oscillating force. The measuring cell oscillates at its own fundamental frequency, which is a function of the mass of the system. During the oscillation, the viscous component of a Newtonian liquid causes, on one hand, a formation of a boundary layer which increases the inertial mass of the resonator and, on the other hand, a damping of the oscillation due to the wall shear stress acting on the resonator. Consequently, these two parasitic effects lead to a lower resonance frequency of the measuring system and, thus, to an inaccurate density measurement. The detection of these influences is performed, in the instruments, by analysing the frequency of harmonics of the oscillation. So, these effects can be corrected by means of algorithms obtained during the calibration of the oscillator with viscous certified reference materials. These measuring instruments were proven to be able to measure density of Newtonian fluids in a wide density, viscosity, and temperature ranges, with an uncertainty better than $0.001 \mathrm{~kg} \cdot \mathrm{m}^{-3}$ [2] when using adequate calibration methods, as the substitution method. Density measurement uncertainty values between $0.010 \mathrm{~kg} \cdot \mathrm{m}^{-3}$ and $0.005 \mathrm{~kg} \cdot \mathrm{m}^{-3}$ can be achieved when regular calibration methods are used, i.e. by means of the used of reference liquids and calibration curves. However, most of the liquids measured by the end-users consist in viscoelastic materials, i.e. materials that, under a certain strain, show simultaneously viscous and elastic behaviour. The viscous portion behaves according to Newton's law of viscosity and the elastic portion to Hooke's law of elasticity.

With the purpose of getting the first insight on how different laboratories handle the density measurements' artefacts caused by the viscoelasticity of non-Newtonian fluids when using oscillation-type density meters, an international comparison was performed, within the scope of the EMPIR Project 17RPT02 rhoLiq [3].

\section{DESCRIPTION OF THE WORK}

This work presents the results of the first international comparison on density of viscoelastic 
samples by oscillation-type densimetry. This comparison involved a set of measurements performed with six oscillators, all borosilicate-made (DMA 4500M, DMA 5000, and DMA 5000M all from Anton Paar), by five participants: five National Metrology Institutes (GUM, Poland; IPQ, Portugal; IMBiH, Bosnia and Herzegovina; PTB, Germany and INM, Moldova) and one density meter manufacturer (Anton Paar, Austria). For this comparison, seven viscoelastic fluids were prepared and distributed among the participants: poly(vinyl alcohol) (PVA) at $0.036 \mathrm{~g} \cdot \mathrm{mL}^{-1}$ and sodium borate

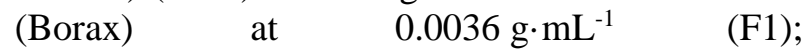
carboxylpolymethylene (Carbomer) solution at $0.15 \mathrm{cg} \cdot \mathrm{g}^{-1} \quad$ (F2); hydroxyethyl-cellulose at $0.5 \mathrm{cg} \cdot \mathrm{g}^{-1}(\mathrm{~F} 3)$; starch dispersion at $3 \mathrm{cg} \cdot \mathrm{g}^{-1}$ (F4); poly(acrylamide-co-diallyldimethylammonium chloride) (P(AAm-co-DADMAC)) solution at $5.5 \mathrm{cg} \cdot \mathrm{g}^{-1}(\mathrm{~F} 5)$; commercial apple (F6) and grape juices (F7) (Table 1).

Each participant followed their own density measuring procedure and have applied corrections to the density meters indications according to the calibration curve obtained with Newtonian reference fluids. These curves allowed to describe the dependence of oscillation damping, in terms of density error, with viscosity of this type of fluids (with no elastic behaviour). Calibration curves with non-Newtonian liquids were not done as viscoelastic reference liquids are not available yet. Therefore, none of the laboratories made corrections due to the elasticity of the samples tested in this comparison. The metrological traceability of density results of each participant is independent. All the measurements were performed at $20^{\circ} \mathrm{C}$ and at ambient pressure.

The reference density value $\rho_{\mathrm{r}}\left(\right.$ at $\left.20^{\circ} \mathrm{C}\right)$ of each test fluid was determined by IPQ and GUM through gravimetric method with the use of pycnometers (Table 1). These tests were performed by substitution weighing method and using the approach described in ISO 2811-1 [4]. Despite of its high uncertainty (when compared with the best uncertainty able to be achieved with oscillation-type density meters, i.e., $0.001 \mathrm{~kg} \cdot \mathrm{m}^{-3}$ [2]), the pycnometry method was chosen to be used as reference as it is a static density measurement method. In this way, the viscoelastic properties of the fluids are not expected to influence the accuracy and precision of these density measurements. Hydrostatic weighing method could be used as reference alternative; however, this method was not yet studied with viscoelastic samples. This corresponds to one of the objectives of the rhoLiq Project [3].

The mechanical properties of the samples, such as complex shear modulus $G^{*}=G^{\prime}+i G^{\prime \prime}$, storage modulus $G^{\prime}$ and loss modulus $G^{\prime \prime}$, under the oscillation frequency $\omega$ (equal to $2 \pi f$, where $f$ stands for frequency) produced during the density measurements in the oscillation-type density meters, usually in the oscillation frequency interval from $1715 \mathrm{~Hz}$ to $1753 \mathrm{~Hz}$, were determined by oscillatory tests with a rheometer (HAAKE Mars III, ThermoScientific) using two different measuring geometries: a cone-plate $\left(\mathrm{C} 35 / 2^{\circ} \mathrm{Ti} \mathrm{L}\right)$ for $\mathrm{P}(\mathrm{AAm}-$ co-DADMAC) and PVA and Borax samples and a concentric-cylinder (CC25 Din Ti) for the remaining samples. The loss or damping factor $\tan \delta$, was calculated as the quotient of the loss $G$ "' and the storage moduli $G^{\prime}$. Thus, this parameter gives the ratio between the viscous and the elastic portion of the viscoelastic deformation.

Table 1: Codification of the test fluids (F\#) and reference density values $\rho_{\mathrm{r}}$, at $20^{\circ} \mathrm{C}$ and ambient pressure, and respective expanded uncertainty values $U \rho_{\mathrm{r}}$ (for $k=2$ ) determined by gravimetric method with a pycnometer

\begin{tabular}{|l|l|l|l|}
\hline F\# & Fluid & $\begin{array}{c}\boldsymbol{\rho}_{\mathbf{r}} \\
/ \mathbf{k g} \cdot \mathbf{m}^{-3}\end{array}$ & $\begin{array}{c}\boldsymbol{U} \boldsymbol{\rho _ { \mathbf { r } }} \\
/ \mathbf{k g} \cdot \mathbf{m}^{-3}\end{array}$ \\
\hline F1 & PVA and Borax & 1008.159 & 0.077 \\
\hline F2 & Carbomer & 998.764 & 0.089 \\
\hline F3 & Hydroxyethyl-cellulose & 999.645 & 0.070 \\
\hline F4 & Starch dispersion & 1008.21 & 0.10 \\
\hline F5 & P(AAm-co-DADMAC) & 1012.139 & 0.086 \\
\hline F6 & Apple juice & 1048.983 & 0.075 \\
\hline F7 & Grape juice & 1068.107 & 0.071 \\
\hline
\end{tabular}

The density values of the test fluids obtained by the oscillation-type density meters of each participant were compared against the reference density values $\rho_{\mathrm{r}}$ (obtained by gravimetric method using a pycnometer) by means of the normalised error $E_{\mathrm{n}}$ statistical analysis [5]. In addition, the mechanical properties of the tested fluids under such oscillation frequency $\omega$ was also correlated with the obtained density deviations.

The measurement uncertainties were performed according to GUM methodology [6].

\section{MECHANICAL CHARACTERISATION OF VISCOELASTIC SAMPLES}

The complex shear modulus, $G^{*}=G^{\prime}+i G^{\prime \prime}$, of the viscoelastic samples were measured in the oscillation frequency range $\omega$, from $0.6 \mathrm{~Hz}$ to $628 \mathrm{~Hz}$, within linear viscoelastic region of the samples, by using a rheometer (HAAKE Mars III, ThermoScientific) with two different measuring geometries: a cone-plate $\left(\mathrm{C} 35 / 2^{\circ} \mathrm{Ti} \mathrm{L}\right)$ for samples $\mathrm{F} 1$ and F5 and a concentric-cylinder (CC25 Din Ti) for the remaining samples. As the range of oscillation frequencies of interest (i.e. the ones produced by the density meter's measuring cell) is higher than the frequency range able to be measured 
by rheometry, an extrapolation methodology was used as described later.

As demonstrated in Figure 1, the storage $G^{\prime}$ and the loss moduli $G^{\prime \prime}$ of all seven samples closely follow an exponential frequency characteristic in the high frequency regime whereas, for low frequencies, considerable deviation from an exponential behaviour is observed. In rheology, viscoelastic materials can be modelled by determination of their response to a stimulus by an arbitrary combination of the properties of an ideal elastic, ideal viscous, and ideal malleable model materials. However, the behaviour modelled in this way still shows differences to the behaviour of real materials, even with complex model bodies. This has several reasons. The Hookean and Newtonian elements assume a linear relationship between stress and deformation, or deformation speed. However, there is often a non-linear relationship. With socalled non-Newtonian fluids, effects such as dilatancy and structural viscosity occur, i.e. the viscosity is no longer a constant, but in turn depends on the rate of deformation. Viscosity can even change with time while the deformation rate remains constant, which is called thixotropy or rheopexy. Finally, the deformation behaviour also depends on external influences, especially temperature. Thus, for the characterisation of the viscoelastic behaviour of the samples, a phenomenological approach was used.

Detailed analysis of the low-frequency dielectric relaxation reveals (Figure 2 (bottom)) that the spectra in the low frequency regime of all samples can be better fitted to a modified power law behaviour given by:

$\ln G^{\prime}=A^{\prime}\left(\ln \omega-C^{\prime}\right)^{m \prime}+B^{\prime}$

$\ln G^{\prime \prime}=A^{\prime \prime}\left(\ln \omega-C^{\prime \prime}\right)^{m^{\prime \prime}}+B^{\prime \prime}$

with $0 \leq m^{\prime} \leq 1$ and $0 \leq m^{\prime \prime} \leq 1$.

In order to obtain quantitative information of the complex viscosity at the resonance frequency of the oscillation-type density meter, the analysis of the complex shear modulus in the high-frequency regime was performed as follows. The storage $G^{\prime}$ and the loss $G^{\prime \prime}$ moduli of all seven samples closely follow an exponential frequency characteristic (Figure 1 and Figure 2 (top)).

In order to prevent values with no physical meaning for the storage modulus $G^{\prime}$, which describes the elastic portion of the complex shear modulus, an upper limit of $G_{\mathrm{S}}=80 \mathrm{GPa}$, the shear modulus of steel, was considered for the fitting function. That means, that it was assumed, that the stiffness of all samples under test do not exceed the stiffness of steel. Thus, the spectra in the high frequency regime for the storage modulus of all samples can be best fitted to: $\ln G^{\prime}=\ln G_{\mathrm{S}}-B e^{-C \omega}$

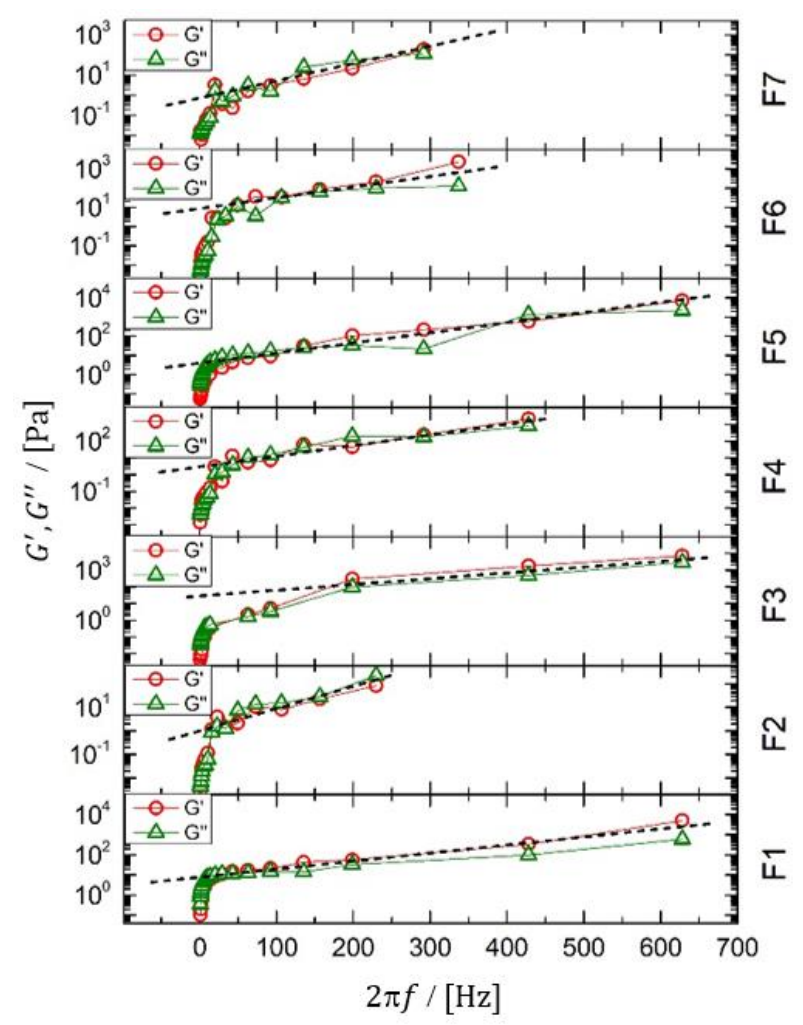

Figure 1: Storage $G^{\prime}$ and loss moduli $G^{\prime \prime}$ as a function of the oscillation frequency $\omega(2 \pi f)$. The black dashed line is a guide to the eye which depicts an exponential behaviour at higher frequencies
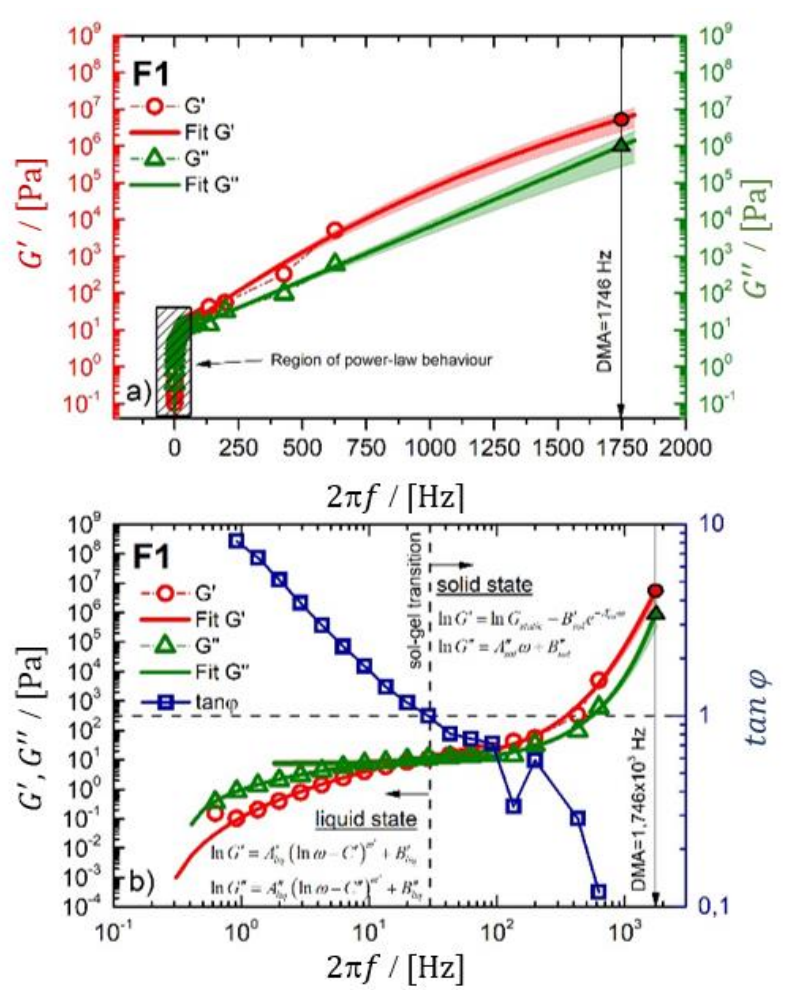

Figure 2: Fits of the storage modulus $G^{\prime}$, loss modulus $G^{\prime \prime}$, and damping factor $\tan \varphi$ spectra against the oscillation frequency $\omega(2 \pi f)$, according to equations (1) to (4), for the sample F1 
The loss modulus $G^{\prime \prime}$ is not limited since it is governed by the viscous part of the material. The viscosity is defined by the relation between shear stress and the velocity gradient perpendicular to the deformation. Hence, the spectra in the high frequency regime for the loss modulus of all samples can be best fitted to:

$$
\ln G^{\prime \prime}=A \omega+C
$$

From the calculated values of $G^{\prime}$ and $G^{\prime \prime}$, the viscous portion, $\eta^{\prime}$ i.e. dynamic viscosity, and the elastic portion, $\eta^{\prime \prime}$, at the oscillation frequency produced in the oscillation-type density meter during the density measurement, were calculated according to,

$$
\eta^{\prime}=\frac{G^{\prime \prime}}{\omega}
$$

and

$$
\eta^{\prime \prime}=\frac{G^{\prime}}{\omega}
$$

The loss or damping factor $\tan \varphi$ was calculated as the quotient of the loss and the storage modulus. Thus, this parameter gives the ratio between the viscous and the elastic portion of the viscoelastic deformation. It is given by:

$\tan \varphi=\frac{G^{\prime \prime}}{G^{\prime}}$

If $\tan \varphi>1$ the sample is in liquid state i.e. showing a behaviour of a viscoelastic liquid, for $\tan \varphi<1$ the sample is in a solid state i.e. showing

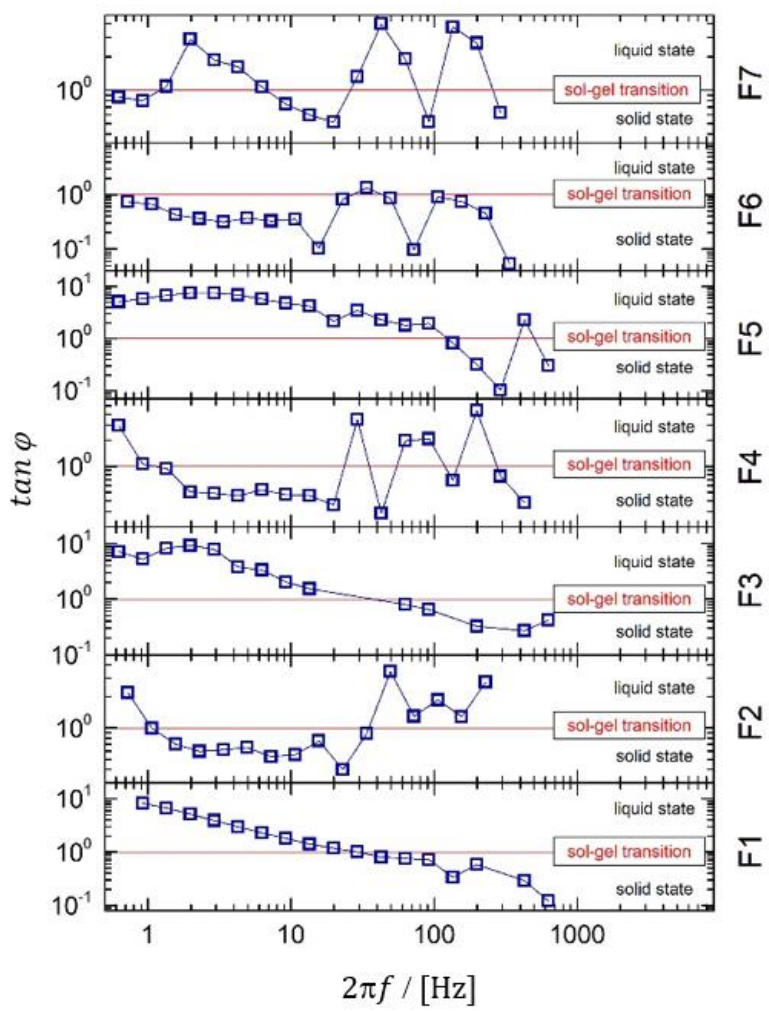

Figure 3: Damping factor $\tan \varphi$ as a function of the oscillation frequency $\omega(2 \pi f)$ for the seven samples tested a behaviour of viscoelastic solid, and for $\tan \varphi=1$ a sol-gel transition will be met, i.e. having a viscoelastic behaviour showing 50:50 ratio of the viscous and elastic portions.

From the analysis of the damping factor $\tan \varphi$ (Figure 3) the transition between the different frequency behaviour of the complex modulus may be related to the sol-gel transition of the material. However, discrepancies exist.

The values of the storage $G^{\prime}$ and loss $G^{\prime \prime}$ moduli and damping factor $\tan \varphi$ of the seven viscoelastic test fluids (F\#) are given in Table 2. In Table 3 are shown the values of the dynamic $\eta$ ' and "elastic" $\eta$ " viscosities of the seven viscoelastic test fluids (F\#) at oscillation frequency $\omega$ of the oscillation-type density meter obtained from the values of Table 2 and equations (5) and (6).

Table 2: Storage $G^{\prime}$ and loss $G^{\prime \prime}$ moduli and damping factor $\tan \varphi$ of the seven viscoelastic test fluids $(\mathrm{F \#})$ at oscillation frequency $\omega$ of the oscillation-type density meter

\begin{tabular}{|c|c|c|c|c|}
\hline F\# & $\begin{array}{c}\boldsymbol{\omega} \\
/ \mathbf{H z}\end{array}$ & $\begin{array}{c}\boldsymbol{G}^{\prime} \\
/ \mathbf{P a}\end{array}$ & $\begin{array}{c}\boldsymbol{G}^{\prime \prime} \\
/ \mathbf{P a}\end{array}$ & $\tan \boldsymbol{\varphi}$ \\
\hline F1 & 1746 & $5.4 \times 10^{6}$ & $9.9 \times 10^{5}$ & 0.18 \\
\hline F2 & 1750 & $2.1 \times 10^{8}$ & $1.6 \times 10^{14}$ & $710^{5}$ \\
\hline F3 & 1750 & $3.0 \times 10^{6}$ & $2.1 \times 10^{7}$ & 6.8 \\
\hline F4 & 1745 & $6.9 \times 10^{7}$ & $5.0 \times 10^{8}$ & 7.2 \\
\hline F5 & 1745 & $1.1 \times 10^{7}$ & $2.5 \times 10^{8}$ & 22 \\
\hline F6 & 1729 & $2.2 \times 10^{8}$ & $1.8 \times 10^{4}$ & $8.0 \cdot 10^{-5}$ \\
\hline F7 & 1721 & $3.5 \times 10^{8}$ & $1.5 \times 10^{8}$ & 0.44 \\
\hline
\end{tabular}

Table 3: Dynamic $\eta$ ' and "elastic" $\eta$ ", viscosities of the

\begin{tabular}{|c|c|c|c|}
\hline F\# & $\begin{array}{c}\omega \\
/ \mathbf{H z}\end{array}$ & $\begin{array}{c}\eta^{\prime} \\
/ \text { Pa·s }\end{array}$ & $\begin{array}{c}\eta^{\prime \prime} \\
\text { / Pa·s }\end{array}$ \\
\hline F1 & 1746 & 568 & 3088 \\
\hline F2 & 1750 & $9 \times 10^{10}$ & $1.2 \times 10^{5}$ \\
\hline F3 & 1750 & $1.2 \times 10^{4}$ & 1727 \\
\hline F4 & 1745 & $2.9 \times 10^{5}$ & $4.0 \times 10^{4}$ \\
\hline F5 & 1745 & $1.4 \times 10^{5}$ & 6400 \\
\hline F6 & 1729 & 10.5 & $1.3 \times 10^{5}$ \\
\hline F7 & 1721 & $8.9 \times 10^{4}$ & $2.0 \times 10^{5}$ \\
\hline
\end{tabular}
seven viscoelastic test fluids (F\#) at oscillation frequency $\omega$ of the oscillation-type density meter

\section{RESULTS OF DENSITY MEASUREMENTS}

The assessment of density deviation values $\delta^{\prime} \rho$ obtained by each laboratory, and respective uncertainties $U_{\delta} \rho$, presented in Table 3, was done by means of the normalised error $E_{\mathrm{n}}$ statistical analysis [5] and are summarised in Table 4. The density values used as reference $\rho_{r}$ are the ones obtained by gravimetric method and shown in Table 1. The missing values of Table 4 are due to some anomalies found in some of the samples. 
Table 3: Summary of the density deviations $\delta \rho$ and respective expanded uncertainties $U_{\delta^{\prime}}(k=2)$, obtained by six laboratories for the seven test fluids (F\#)

\begin{tabular}{|c|c|c|c|c|c|c|}
\hline \multirow{3}{*}{ F\# } & \multicolumn{2}{|c|}{ GUM } & \multicolumn{2}{|c|}{ IPQ } & \multicolumn{2}{|c|}{ IMBiH } \\
\hline & $\delta^{\prime} \rho$ & $U_{\delta^{\prime} \rho}$ & $\delta^{\prime} \rho$ & $U_{\delta \rho}^{\prime}$ & $\delta^{\prime} \rho$ & $U_{\delta^{\prime} \rho}$ \\
\hline & \multicolumn{6}{|c|}{$/ 10^{-2} \mathrm{~kg} \cdot \mathrm{m}^{-3}$} \\
\hline F1 & -2.3 & 7.8 & 2.3 & 9.4 & -3.8 & 8.3 \\
\hline $\mathrm{F} 2$ & -7.6 & 9.1 & -7.2 & 9.1 & - & - \\
\hline F3 & 9.0 & 7.2 & 10.8 & 8.9 & -30.2 & 20.8 \\
\hline $\mathrm{F} 4$ & - & - & 11.0 & 10.3 & - & - \\
\hline F5 & -17.7 & 9.9 & - & - & - & - \\
\hline F6 & 3.6 & 7.7 & 1.3 & 7.8 & -0.3 & 8.2 \\
\hline F7 & 4.6 & 7.2 & 5.8 & 7.4 & -10.8 & 14.4 \\
\hline \multirow{3}{*}{ F\# } & \multicolumn{2}{|c|}{ INM-MD } & \multicolumn{2}{|c|}{ PTB } & \multicolumn{2}{|c|}{ Anton Paar } \\
\hline & $\delta^{\prime} \rho$ & $U_{\delta^{\prime} \rho}$ & $\delta^{\prime} \rho$ & $U_{\delta^{\prime} \rho}$ & $\delta^{\prime} \rho$ & $U_{\delta^{\prime} \rho}$ \\
\hline & \multicolumn{6}{|c|}{$/ 10^{-2} \mathrm{~kg} \cdot \mathrm{m}^{-3}$} \\
\hline F1 & -8.0 & 8.3 & -8.9 & 7.8 & -7.2 & 9.4 \\
\hline $\mathrm{F} 2$ & -11.4 & 9.8 & -13.4 & 9.1 & -9.0 & 10.2 \\
\hline F3 & 7.1 & 7.7 & 3.7 & 7.1 & 9.1 & 8.4 \\
\hline F4 & 5.8 & 10.6 & 9.4 & 10.2 & 8.6 & 11.1 \\
\hline F5 & -50.2 & 9.1 & -18.6 & 8.8 & 5.9 & 21.9 \\
\hline F6 & 12.5 & 8.4 & -1.1 & 7.7 & - & - \\
\hline F7 & -4.4 & 7.7 & -2.7 & 7.2 & 2.4 & 11.4 \\
\hline
\end{tabular}

Table 4: Summary of the statistical analysis of the normalised error $E_{\mathrm{n}}$ obtained by the participant laboratories for each test fluid (F\#)

\begin{tabular}{|c|c|c|c|c|c|c|}
\hline \multirow{2}{*}{ F\# } & GUM & IPQ & IMBiH & INM & PTB & $\begin{array}{c}\text { Anton } \\
\text { Paar }\end{array}$ \\
\cline { 2 - 7 } & \multicolumn{7}{|c|}{$\boldsymbol{E}_{\mathbf{n}}$} \\
\hline F1 & 0.30 & 0.25 & 0.46 & 0.96 & 1.14 & 0.77 \\
\hline F2 & 0.83 & 0.79 & - & 1.17 & 1.47 & 0.89 \\
\hline F3 & 1.26 & 1.21 & 1.45 & 0.92 & 0.52 & 1.08 \\
\hline F4 & - & 1.06 & - & 0.55 & 0.92 & 0.77 \\
\hline F5 & 1.79 & - & - & 5.52 & 2.11 & 0.27 \\
\hline F6 & 0.47 & 0.17 & 0.03 & 1.49 & 0.15 & - \\
\hline F7 & 0.63 & 0.78 & 0.75 & 0.57 & 0.38 & 0.21 \\
\hline
\end{tabular}

The weighted density mean values $\overline{\rho_{W}}$ (with respective expanded uncertainty $U \overline{\rho_{W}}$ ) (Table 5) and weighted relative density mean values $\delta^{\prime} \overline{\rho_{W}}$ (with respective relative expanded uncertainties $\left.U_{\delta}, \overline{\rho_{W}}\right)$ (Table 5), were calculated having in account only the satisfactory results (i.e., with $E_{\mathrm{n}}<1$ ) (Table 6).

For the calculation of the relative expanded uncertainties of the weighted relative density mean values $U_{\delta}, \overline{\rho_{W}}$ no correlation was considered among the values as each laboratory has its own traceability chain. This uncertainty also includes the contribution of the uncertainty due to the heterogeneity of the samples.

Table 6 demonstrates that, on average, $38 \%$ of the measurements of these viscoelastic samples presented unsatisfactory measurement results, meaning that the laboratories are underestimating Table 5: Weighted density mean values $\overline{\rho_{W}}$, and respective expanded uncertainty $U \overline{\rho_{W}}(k=2)$ of unsatisfactory results

\begin{tabular}{|c|c|c|}
\hline F\# & $\begin{array}{c}\overline{\boldsymbol{\rho}_{\boldsymbol{W}}} \\
/ \mathbf{k g} \cdot \mathbf{m}^{-3}\end{array}$ & $\begin{array}{c}\boldsymbol{U} \overline{\boldsymbol{\rho}_{\boldsymbol{W}}} \\
/ \mathbf{k g} \cdot \mathbf{m}^{-\mathbf{3}}\end{array}$ \\
\hline F1 & 1008.12 & 0.19 \\
\hline F2 & 998.67 & 0.21 \\
\hline F3 & 999.70 & 0.10 \\
\hline F4 & 1008.29 & 0.18 \\
\hline F5 & 1012.20 & 0.22 \\
\hline F6 & 1048.99 & 0.16 \\
\hline F7 & 1068.09 & 0.24 \\
\hline
\end{tabular}

Table 6: Summary of the weighted relative density deviation mean values $\delta^{\prime} \overline{\rho_{W}}$, respective relative expanded uncertainties $U_{\delta}, \overline{\rho_{W}}(k=2)$, and frequency $f$ of unsatisfactory results (i.e., with $E_{\mathrm{n}}>1$ ) for each fluid (F\#)

\begin{tabular}{|c|c|c|c|}
\hline F\# & $\begin{array}{c}\boldsymbol{\delta}^{\prime} \overline{\boldsymbol{\rho}_{\boldsymbol{W}}} \\
\boldsymbol{/ \%}\end{array}$ & $\begin{array}{c}\boldsymbol{U}_{\boldsymbol{\delta}}, \overline{\boldsymbol{\rho}_{\boldsymbol{W}}} \\
\boldsymbol{/ \%}\end{array}$ & $\begin{array}{c}\boldsymbol{f}\left(\boldsymbol{E}_{\mathbf{n}}>\mathbf{1}\right) \\
/ \boldsymbol{\%}\end{array}$ \\
\hline F1 & -0.004 & 0.019 & 17 \\
\hline F2 & -0.010 & 0.021 & 40 \\
\hline F3 & 0.005 & 0.010 & 67 \\
\hline F4 & 0.008 & 0.018 & 25 \\
\hline F5 & 0.006 & 0.022 & 80 \\
\hline F6 & 0.001 & 0.015 & 20 \\
\hline F7 & -0.002 & 0.022 & 17 \\
\hline
\end{tabular}

the uncertainty and/or the corrections applied to density indications to compensate measurement artefacts due to samples' viscosity and elasticity are not sufficiently established due to the lack of previous knowledge on this subject. However, the establishment of a calibration curve for viscoelastic fluids, due the great number of possible variations on the viscous and elastic portion, is not an easy task to achieve. Another point that should be taken into consideration is the stability and homogeneity of the samples, since they were not all measured at the same time by the laboratories and the long-term stability and the stability under transportation condition was not tested, and therefore not considered. In Table 6 , the high frequencies of unsatisfactory values $\left(f\left(E_{\mathrm{n}}>1\right)\right)$ presented in fluids F2, F3 and F5 can be a proof of this.

Previous unpublished investigations, of Newtonian liquids, showed a linear dependence of density deviations on the squared root of dynamic viscosity $\left(\sqrt{\eta^{\prime}}\right)$ up to viscosity of $\sim 0.3 \mathrm{~Pa} \cdot \mathrm{s}$ reaching a plateau of maximum density deviation value achieved when the thickness of the fluid boundary layer was equal to the inner diameter of the oscillator cell, where the density deviation does not longer depends on the squared root of dynamic viscosity $\left(\sqrt{\eta^{\prime}}\right)$.

However, for the lowest value of dynamic viscosity of the tested viscoelastic samples is $10 \mathrm{~Pa} \cdot \mathrm{s}$, meaning that the mentioned plateau was already achieved, so considering the weighted relative density deviation mean values $\delta^{\prime} \overline{\rho_{W}}$ 
(Table 6 and Figure 4) can be seen that it was not found any dependence of density deviation with the squared root of dynamic viscosity $\left(\sqrt{\eta^{\prime}}\right)$.

The influence of viscoelastic properties of a fluid on the resonance frequency of the oscillation-type density meter is given by, on the one hand, that additional harmonic forces act on the oscillator due to the elastic portion of the shear modulus of the fluid. This leads to an apparent increase of the spring constant of the oscillator. On the other hand, the viscous portion of the shear modulus of the fluid increases the inert mass of the oscillator due to the movement of fluid layers in the boundary layer which is in phase with the oscillator.

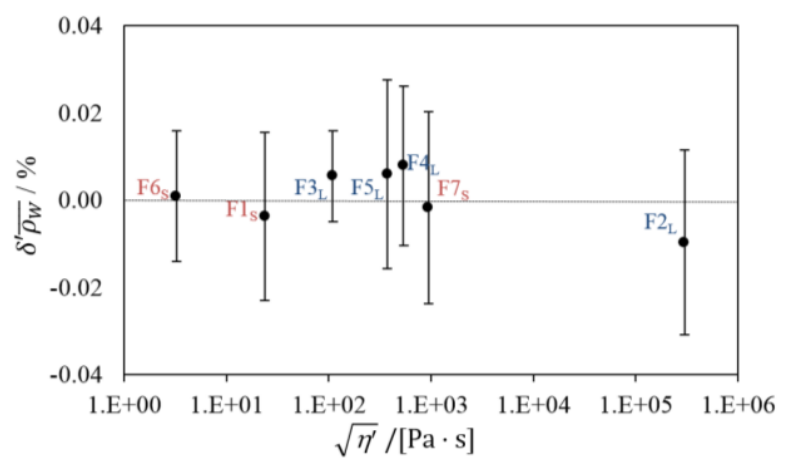

Figure 4: Weighted relative density mean values $\delta^{\prime} \overline{\rho_{W}}$ and respective uncertainties (vertical bars), obtained for the seven tested viscoelastic fluids (F\#), against the squared root of dynamic viscosity $\left(\sqrt{\eta^{\prime}}\right)$ of the fluids. Legend: $\mathrm{S}$ or $\mathrm{L}-$ sample at solid ( $\mathrm{S}$ in red) or liquid ( $\mathrm{L}$ in blue) state at the oscillation frequency produced in the oscillation-type density meter

\section{SUMMARY}

This paper shows the results of the first international comparison on density of viscoelastic samples by oscillation-type densimetry, allowing to conclude about the degree of equivalence of density measurements of this type of samples with this measuring method, by experts on this metrology field.
From the results it seems to be possible to measure density of viscoelastic samples by means of oscillation-type density meters with an uncertainty between $0.10 \mathrm{~kg} \cdot \mathrm{m}^{-3}$ and $0.25 \mathrm{~kg} \cdot \mathrm{m}^{-3}$. The lower limit of this uncertainty interval was limited by the uncertainty of the method used as reference (pycnometry with a measurement uncertainty of $\sim 0.10 \mathrm{~kg} \cdot \mathrm{m}^{-3}$ ), meaning that these instruments may be able to reach lower uncertainties if compare with higher precision density measurement methods such as hydrostatic weighing. Further investigations will be done on this topic under the scope of the EMPIR project "17RPT02-rhoLiq".

This work was developed under the framework of the EMPIR project "17RPT02-rhoLiq" which is carried out with funding of European Union under the EMPIR. The EMPIR is jointly funded by the EMPIR participating countries within EURAMET and the European Union.

\section{REFERENCES}

[1] H. Stabinger, "Density Measurement using modern oscillating transducers", South Yorkshire Trading Standards Unit, Sheffield, 1994.

[2] H. Schmidt, H. Wolf, E. Hassel, "A method to measure the density of seawater accurately to the level of $10^{-6}$ ", Metrologia, vol. 53, no. 2, p. 770, 2016.

[3] A. Furtado, J. Pereira, M. Schiebl et al., "Establishing traceability for liquid density measurements in Europe: 17RPT02-rhoLiq a new EMPIR joint research project", Journal of Physics: Conference Series, vol. 1065, no. 8, 082013, IOP Publishing, 2018.

[4] ISO 2811-1, "Paints and varnishes - Determination of density - Part 1: Pycnometer method", 2016.

[5] ISO 13528, "Statistical methods for use in proficiency testing by interlaboratory comparison", 2015.

[6] JCGM 100, "Evaluation of measurement data Guide to the expression of uncertainty in measurement", BIPM, 2008. 